\title{
Pure Obstructive Sleep Apnea Syndrome and Erectile Dysfunction
}

\author{
Cenk Gürbüz'1, Hacer Kuzu Okur², Selamettin Demir'1, Salih Ordu'1, Turhan Caşkurlu \\ 'Department of Urology, Istanbul Göztepe Training and Research Hospital, İstanbul, Turkey \\ ${ }^{2}$ Department of Chest Diseases, Süreyyapaşa Chest Diseases Training and Research Hospital, Istanbul, Turkey
}

\begin{abstract}
Objective: The aim of this study is to investigate the existence of erectile dysfunction in patients with obstructive sleep apnea syndrome (OSAS) in which the other possible causes of erectile dysfunction were eliminated.

Material and Methods: The study group consisted of 24 patients diagnosed as OSAS with polysomnographic evaluation, and 15 non-apneic controls (mean age; $41.0 \pm 8.8$ and $42.3 \pm 7.9$ year respectively) whose comorbidities which might be associated with erectile dysfunction were excluded. Daytime sleepiness was evaluated by Epworth Sleepiness Scale (ESS) and measurement of erectile function was performed by International Index of Erectile Function.

Results: The rate of erectile dysfunction in OSAS and control groups were $54.2 \%$ and $33.3 \%$ respectively ( $p=0.204$ ). The difference between mean erectile function scores of patient and control groups was non-significant $(26.1 \pm 4.5$ and $26.3 \pm 4.3$ respectively, $p=0.900)$. There was no correlation between erectile function scores and apnea hypnoea index $(r=-0.140 ; p=0.395)$.
\end{abstract}

Conclusion: Findings obtained from this study suggest that the high incidence of erectile dysfunction reported in OSAS patients seems to be related with concomitant comorbidities such as diabetes, atherosclerosis and neuroendocrine disorders rather than sleep apnea.

Key Words: Erectile dysfunction, sleep apnea, international index of erectile function, polysomnography

Received: 02.07.2010

Accepted: 16.12 .2010

\section{Introduction}

Obstructive sleep apnea syndrome (OSAS) is a condition characterized by repetitive episodes of airflow limitation lasting at least 10 seconds (1). The prevalence of OSAS based on the current definition is rising worldwide. OSAS has become a common and serious problem and also has a negative effect on the quality of life, (2) especially in overweight patients. It is usually associated with a number of different systemic complications such as hypertension, hyperlipidemia, cardiovascular diseases and increased risk of stroke (3). The mechanism underlying these complications is likely to be multifactorial, involving recurrent intermittent hypoxia and increased sympathetic activation (4).

Erection is an event that involves the interaction of psychological, neurological, endocrinological and vascular systems. Any impairment in this cooperation may cause erectile dysfunction (ED). Erectile dysfunction as a symptom of OSAS has been previously reported in the literature $(5,6)$. Although the etiologic association between OSAS and ED is controversial, sexual problems are common among men with sleep apnea/hypopnea. An incidence of $48 \%$ of sexual problems has been reported among men aged 25-65 year with sleep apnea (7). The mechanism underlying ED in patients with OSAS remains unclear. However, nasal continuous positive airway pressure (CPAP), which is the gold standard in treatment of OSAS patients, has been found effective in improving sexual performance (8).

Erectile dysfunction may be a consequence of frequently associated comorbidities such as obesity and vascular disease in this patient population, rather than being secondary to OSAS (9). We hypothesized that OSAS may not be the primary etiology of ED. In this prospective study, we aimed to investigate the status of erectile function in OSAS patients without any other comorbidity which may lead to erectile dysfunction, and also to correlate the apnea hypopnea index and erectile function scores.

\section{Materials and Methods}

\section{Study population}

This is a prospective clinical study which was conducted between August 2007 and April 2008 after the approval of the local Ethics Committee. The study population consisted of men who were admitted to the sleep clinic for an initial evaluation and had symptoms consistent with sleep apnea as defined by a sleep specialist. Diagnosis of OSA was based on the clinical history, Epworth Sleepiness Scale (ESS) scores (9) and polysomnography (PSG) findings. The diagnosis of OSAS was confirmed by an apnea-hypopnea index (AHI) of $>10$ episodes/hr of sleep during a full-night PSG performed by a sleep specialist. 
Exclusion criteria were patients with cardiovascular diseases, hypertension, deep vein thrombosis, peripheral vascular disease, hyperlipidemia, connective tissue disorders, and metabolic or neurologic disorders inducing peripheral neuropathy (e.g., diabetes mellitus). Patients who were already diagnosed with ED or were treated with medications that affect erectile function (e.g., $\beta$-blockers, histamine type 2 receptor-blockers, or PDE-5 inhibitors) were also excluded. The existence of the confounding risk factors were excluded by routine history taking and medical documentation, full physical examination including blood pressure measurement and laboratory assessment including total cholesterol, triglyceride, HDL, LDL, VDL, and blood sugar level.

Of the 135 patients enrolled, 24 met the eligibility criteria in the OSAS group. The control group $(n=15)$ included men with symptoms suggestive of OSAS snoring, apnea, daytime sleepiness (but diagnosed with simple snoring), and an $\mathrm{AHI}$ $<10$. All volunteers provided written informed consents before participation.

\section{Polysomnography}

All patients and controls underwent full-night PSG (CometGrass Technologies Astro-Med, USA). Standard PSG montages were used as follows: 4-channels electroencephalography (EEG, C4-A2,C3-A1, O2-A1, O1-A2), left and right electrooculography (EOG), submental electromyography (EMG), a nasal cannula to record nasal pressure and thermistor to monitor nasal and oral airflow, left and right anterior tibialis movement sensors, respiratory effort by thoraco-abdominal belts, electrocardiography (ECG), finger pulse oximetry, a neck microphone for recording snoring, a sensor on the thoracic belt to record the body posture. PSG recordings were continued for at least 6 hours.

Sleep stage and respiratory event scorings were based on the standard criteria $(10,11)$. Apnea was defined as complete cessation of airflow lasting at least 10 seconds, while hypopnea was defined as a decrement in air flow, if it was associated with either arousal (defined as the appearance of 3 seconds of an alpha rhythm on EEG channels or an increment in the submental EMG signal) or oxygen desaturation of $\geq 3 \%$ (12). $\mathrm{AHI}$ was calculated by dividing the total number of apnea and hypopnea events by the total sleep time. The minimal oxygen saturation was determined as the lowest saturation value associated with a respiratory event.

\section{Assessment of erectile dysfunction and quality of life}

The scores of the International Index of Erectile Function (IIEF) and Quality of Life scale (SF-36) were recorded. IIEF was divided into 5 domains including erectile function, intercourse satisfaction, orgasm, sex drive and overall satisfaction. The erectile function domain consists of six items (questions 1-5 and 15), with a maximal score of 30 and a minimal score of 6. Each question is scored by a 5-point Likert scale, with 5 representing the best function. A total score of $<26$ is indicative of ED. The degree of ED is estimated as a score of 17-25, $11-16$, and $<10$ representing mild, moderate, and severe, respectively (13). The evaluation by the urologist confirmed the presence of ED lasting for at least 6 months. SF-36 is a multi- purpose, short-form health survey with only 36 questions. It yields an 8-scale profile of functional health and well-being scores, psychometrically-based physical and mental health summary measures and a preference-based health utility index. Each scale is directly transformed into a 0-100 scale on the assumption that each question carries equal weight. The eight domains are; vitality, physical function, bodily pain, general health, role physical, role emotional, social function and mental health (14). In this study, all subjects completed the Turkish version of SF-36, which was validated by Kocyigit (15) and the IIEF questionnaire, which was validated in Turkish (16).

Patient interviews were performed by an urologist with residency training and an urologist with specialty training in sexual dysfunction. Diagnosis of ED was made at the end of a structured interview. The urologist was unaware of the severity of the patient's sleep problem.

\section{Body mass index measurement}

The body mass index (BMI) is a simple index of weight-forheight defined as the weight in kilograms divided by the square of the height in meters $\left(\mathrm{kg} / \mathrm{m}^{2}\right)$. BMl categories are defined based on the following criteria; underweight, <18.5; normal weight, 18.5-24.9; overweight, 25-29.9; obese, $\geq 30$ (17).

\section{Statistical analysis}

Statistical Package for Social Science (SPSS) for Windows Version 16.0 software (SPSS, Inc, Chicago, IL, USA) was used. All comparable data were summarized using descriptive statistics (median and standard deviation) and the Mann-Whitney $U$ test for two unrelated variables; a Chi-square test was used for quantitative data and the Pearson correlation test for variable intervals or ratios. $p<0.05$ was set as statistically significant.

\section{Results}

The characteristics of patient and control groups including age, BMI, PSG parameters, and IIEF scores were presented in Table 1. AHI and oxygen desaturation indexes were significantly higher in OSA patients. OSA and control groups showed similar erectile properties measured by IIEF scores. Thirteen $(54.2 \%)$ out of 24 patients with OSA had variable degrees of $E D$, compared with $5 / 15$ (33.3\%) of controls ( $p=0.204)$. None of the patients had severe ED in either group. The number of patients with mild ED in OSA and control groups was 14/24 and $7 / 15$, respectively, while the number of patients with moderate ED was $10 / 24$ and 8/15, respectively.

The mean BMI value was similar in both groups $(p=0.140)$. The number of overweight patients was 12 in both groups, while the number of obese patients was 4 and 11 in OSA and control groups.

The scale profile of functional health and well-being scores of SF-36, which contains 8 domains, were similar in the OSA and control groups $(p>0.05)$. There was no correlation between the IIEF scores and oxygen saturation level, AHI, and apnea index (Al) in the OSA group (Table 2) or between ED and $\mathrm{AHI}, \mathrm{Al}$ and the oxygen desaturation index (ODI). However, ED was significantly correlated with the rapid eye movement (REM) sleep (Table 3). 
Table 1. Demographic, anthropometric, polysomnographic characteristics and erectile function scores of obstructive sleep apnea and control groups

\begin{tabular}{|lccc|}
\hline & $\begin{array}{c}\text { OSA } \\
(\mathbf{n}=\mathbf{2 4})\end{array}$ & $\begin{array}{c}\text { Controls } \\
(\mathbf{n}=15)\end{array}$ & $\mathbf{p}$ \\
\hline Age (years) & $41.0 \pm 8.8$ & $42.3 \pm 7.9$ & 0.663 \\
BMI (kg/m²) & $30.0 \pm 4.0$ & $28.0 \pm 3.7$ & 0.140 \\
AHI (event per hour) & $43.7 \pm 22.2$ & $2.9 \pm 2.6$ & $<0.001$ \\
Epwort Sleep Scale (ESS) & $10.9 \pm 6.7$ & $7.3 \pm 6.3$ & 0.242 \\
Erectile function & $26.1 \pm 4.5$ & $26.3 \pm 4.3$ & 0.900 \\
Lowest SaO (\%) & $76.9 \pm 11.9$ & $87.2 \pm 3.7$ & $<0.001$ \\
Longest apnea (s) & $61.7 \pm 28.7$ & $29.7 \pm 15.1$ & $<0.001$ \\
Stage REM (\%) & $14.8 \pm 6.1$ & $19.6 \pm 5.4$ & 0.017 \\
Al (event per hour) & $36.7 \pm 23.6$ & $2.1 \pm 2.3$ & $<0.001$ \\
AHI (SUPIN) & $40.2 \pm 28.2$ & $4.1 \pm 1.3$ & $<0.001$ \\
AHI (REM) & $37.5 \pm 24.5$ & $3.9 \pm 4.6$ & $<0.001$ \\
ODI (hour) & $45.6 \pm 23.6$ & $5.1 \pm 4.9$ & $<0.001$ \\
REM latency & $150.0 \pm 71.7$ & $107.7 \pm 66.7$ & 0.074 \\
Stage 1 (\%) & $10.0 \pm 7.6$ & $6.3 \pm 4.8$ & 0.102 \\
Stage 2 (\%) & $52.0 \pm 12.8$ & $45.5 \pm 13.3$ & 0.135 \\
Stage 3 (\%) & $20.4 \pm 11.2$ & $26.9 \pm 12.8$ & 0.106 \\
\hline BMI: Body Mass Index, AHI: Apnea Hypopnea Index, Al: Apnea Index, ODI: & & & \\
\hline Oxygen Desaturation Index & & & \\
\hline
\end{tabular}

Table 2. Correlation between erectile function and PSG parameters

\begin{tabular}{|lcc|}
\hline & $\mathbf{r}$ & $\mathbf{p}$ \\
\hline $\mathrm{AHI}$ & -0.140 & 0.395 \\
$\mathrm{ESS}$ & -0.181 & 0.271 \\
Lowest $\mathrm{SaO}_{2}$ & 0.210 & 0.200 \\
Longest apnea(s) & -0.029 & 0.859 \\
Stage REM (\%) & -0.370 & 0.020 \\
$\mathrm{Al}$ & -0.215 & 0.188 \\
AHISupine & -0.228 & 0.168 \\
AHIREM & -0.076 & 0.647 \\
ODI & -0.115 & 0.488 \\
REM latency & -0.020 & 0.902 \\
\hline AHI: apnea hypopnea index, ESS: Epworth sleepiness score, Al: apnea in- \\
dex, AHI: Supine, apnea hyponea index during supine position, AHIREM: \\
apnea hypopnea index during REM sleep, ODI: oxyhemoglobin desatura- \\
tion index
\end{tabular}

Table 3. The relation between REM and Erectile Function

\begin{tabular}{|ccccc|}
\hline & $\begin{array}{c}\text { ED } \\
\text { patients }\end{array}$ & $\begin{array}{c}\text { Patients } \\
\text { without ED }\end{array}$ & Total & p \\
\hline REM\% $\leq 20$ & OSA (8) & OSA (9) & & \\
& Non OSA (1) & Non OSA (8) & 26 & 0.041 \\
REM\% $>20$ & OSA (5) & OSA (2) & & \\
& Non OSA (4) & Non OSA (2) & 13 & \\
\hline
\end{tabular}

\section{Discussion}

The main finding of this study was that erectile dysfunction scores and respiratory parameters such as apnea hypopnea index and desaturation level were not significantly correlated in OSAS patients having no other comorbidities. A negative correlation between sleep apnea and penile rigidity has been mentioned in previous studies, but most patients also present with hypertension, diabetes and other cardiac risk factors (3). Although OSA is associated with ED, it is unknown whether this association is maintained in the presence of other risk factors for ED. In the present study, we eliminated factors affecting erectile function. Since OSA is related to, or has a potential to cause ED, it is difficult to eliminate these factors in the OSA group. To the best of our knowledge, strict exclusion criteria were not employed in the previous studies and this is the first study comprised of isolated OSA patients without any other manifest comorbidities.

Conflicting data exist regarding the relationship between OSA and ED. Several studies have reported a high prevalence of ED in patients with OSA $(4,18)$. Fanfulla et al. (19) have suggested that the underlying mechanism of this association might be mediated by hypoxia-induced occult nerve dysfunction. There are a few studies that do not support the notion that sleep related breathing disorders are involved in an increased prevalence of erectile impotence (9). Based on the above mentioned considerations and the results of the current study, we suggest that OSA may not be the primary etiology of ED.

Vasculogenic, neurogenic, hormonal and psychogenic factors may contribute to the pathogenesis of ED in OSA. Alterations in the nitric oxide cyclic guanosine monophosphate pathway may be one of the other mechanisms involved. Psychological factors, including decreased libido and mood changes associated with sleep fragmentation, may be similarly associated $(2,20)$. Sleep fragmentation itself, irrespective of intermittent hypoxia, is known to affect similar physiologic consequences, including sympathetic activation. Shin et al. (2) demonstrated that total sleep time is longer in the OSAS group, but sleep and REM latency are similar in the OSA and control groups. Their analysis revealed a nonsignificant difference between ED scores and sleep parameters. With respect to sleep architecture, our results were consistent with the above-mentioned literature.

OSA is usually worse in REM sleep than non-rapid eye movement (NREM) sleep $(21,22)$. Series et al. (22) demonstrated a higher reduction in $\mathrm{SaO}_{2}$ after obstructive apnea during REM sleep than during NREM sleep. Findley (21) concluded that sleep apnea is longer during REM sleep than NREM sleep in patients with OSAS, whereas hypoxemia is greater during REM sleep than NREM sleep in subjects with and without OSAS. In the current study, increased REM percentage had a negative effect on erectile function. Correlation analysis between erectile function and PSG findings revealed that the percentage of REM is the only factor significantly correlated with erectile function. A subset analysis suggested ED was correlated with REM stage $(p=0.041)$. The association between ED and REM stage was significantly correlated in the control group $(p=0.036)$ but it was not significantly correlated 
with the OSA group $(p=0.06)$. Due to the limited number of patients in our study group, subclassification of the groups made the statistical power low. We could not show a significant statistical difference in OSA patients, but ED appears to be correlated with REM. A REM sleep percentage $<20 \%$ of the total sleep time seems to be protective for erectile function. The difference in REM quality may well be secondary to psychiatric causes of ED such as depressiveldysthymic disorders. It was reported that the REM stage is longer in this group $(23,24)$. We did not focus on this secondary issue and did not screen for depression. During sleep, every REM period is normally associated with an erection. Since most REM periods occur towards the morning, awaking in the morning from REM sleep with an erection is a common phenomenon (25). However, morning erections were not evaluated in the present study and no nocturnal penile tumescence test was performed to differentiate the possible etiology.

OSAS is common in obese patients and may be associated with metabolic disorders, which are well known causes of ED. It was found that men with a BMI $>28.7$ carry a $30 \%$ higher risk for ED than those with a normal BMI of 25 (26). The mean BMI of the control and OSA groups was similar in the current study. The number of obese patients in the OSA group was higher than the control group (12 vs 4 ) where the remaining patients in both groups were overweight (12 vs 11) which may explain the higher proportion of ED among patients with OSA than the control group (54.2\% vs $33.3 \%$ ).

There are a limited number of studies that used a validated instrument for the assessment of erectile function and OSA. The current study was conducted to evaluate the correlation between OSA and ED, using the IIEF and ESS scores. The diagnosis of OSA was also confirmed with PSG. Although some authors noted an association between OSA and quality of life, different studies produced conflicting results $(27,28)$. These studies relied upon general health questionnaires, such as the SF-36 and satisfaction with life scale to evaluate quality of life. The limitation of SF-36 is that it is not a disease-specific quality of life questionnaire. Therefore, the results may not have addressed issues specifically related to OSAS. The Calgary Sleep Apnea Quality of Life Index (SAQLI) identifies conditions or factors that are specific to patients with sleep apnea. However, there is no Turkish-validated version of SAQLI. In the current study, in which participants were assessed using the SF-36 questionnaire, the total score of SF-36 and each subscale of SF-36 were similar between the isolated OSA and control groups. These findings suggested that the presence of isolated OSA may not be an important factor determining the health-related quality of life; however, we believe that utilizing the SAQLI would be more reliable in this regard.

Previous studies determined that IIEF is readily selfadministered in research or clinical settings due to the high sensitivity and specificity values $(13,16)$. IIEF was used to identify patients with ED in the present study. The items of IIEF were completed with a supervisor and an additional interview was performed by an urologist, in an effort to eliminate possible problems in understanding the questionnaire. Teloken et al. (29) used an ESS score of 10 as the cut-off for diagnosing OSA, and correlated between the severity of ESS and
ED. In the present study, an ESS score $>10$ was considered to be consistent with OSA and the diagnosis was confirmed by PSG. ESS measures the daytime sleepiness and drowsiness and is not specific to OSA. In the current study, there was no correlation between the IIEF and ESS scores. Margel et al. (30) have correlated severe OSA with ED, in all dimensions they assessed. This correlation was dramatic from both the apnea and the erectile function perspectives. Mild or moderate OSAS was only partially associated with ED, whereas severe OSAS was clearly related to ED. In the present study, the severity of OSA was also evaluated via the AHI. However, there was nonsignificant evidence of an increased risk for ED in patients with an $\mathrm{AHI}$ score $>30$ and $<30$ or with an $\mathrm{AHI}$ score $>15$ and $<15$. In evaluating a patient with complaints of impotence, OSA should be considered questionable. Association between milder forms of sleep disturbances and sexual problems was investigated by Hanak et al. (31) whodivided the subjects into categories of heavy, moderate and none/ mild snoring. There was a nonsignificant difference in erectile function assessment across snoring categories. In our study, we did not address the relation between milder forms of sleep apnea and sexual problems.

The major limitation of this study is the low number of patient population. To prevent conflicting results, we eliminated known risk factors for ED in patients with OSA, and patients with sleep apnea were referred to as isolated OSA, but there are some other factors such as life styleand exercising, which should also be considered. The present study failed to show a significant correlation between isolated obstructive sleep apnea syndrome and erectile dysfunction. In previous studies, the increased prevalence of ED in OSA patients may be attributed to the additional risk factors, which are frequently encountered in this group. The existence of the confounding risk factors should be further investigated in the OSA group. There may be a correlation between REM sleep and erectile function.

\section{Conflict of Interest}

No conflict of interest was declared by the authors.

\section{References}

1. Malhotra A, White DP. Obstructive sleep apnea. Lancet 2002;360:237-45. [CrossRef]

2. Shin HW, Rha YC, Han DH, Chung S, Yoon IY, Rhee CS, et al. Erectile dysfunction and disease-specific quality of life in patients with obstructive sleep apnea. Int J Impot Res 2008;20:549-53. [CrossRef]

3. Peker Y, Hedner J, Norum J, Kraiczi H, Carlson J. Increased incidence of cardiovascular disease in middle-aged men with obstructive sleep apnea: a 7-year follow-up. Am J Respir Crit Care Med 2002;166:159-65. [CrossRef]

4. Lavie $P$, Herer P, Hoffstein V. Obstructive sleep apnea syndrome as risk factor for hypertension; population study. $\mathrm{Br}$ Med $\mathrm{J}$ 2000;320:479-82. [CrossRef]

5. Seftel AD, Strohl KP, Loye TL, Bayard D, Kress J, Netzer NC. Erectile dysfunction and symptoms of sleep disorders. Sleep 2002;25:643-7.

6. Hirshkowitz M, Karacan I, Arcasoy MO, Acik G, Narter EM, Williams RL. Prevalence of sleep apnea in men with erectile dysfunction. Urology 1990;36:232-4. [CrossRef] 
7. Guilleminault C, Eldridge FL, Tilkian A, Simmons FB, Dement WC. Sleep apnea syndrome due to upper airway obstruction. A review of 25 cases. Arch Intern Med 1977;137:296-300. [CrossRef]

8. Taskin U, Yigit O, Acioglu E, Arıcıgil M, Toktas G, Guzelhan Y. Erectile dysfunction in severe sleep apnea patients and response to CPAP. Int J Impotence Res 2010;22:134-9. [CrossRef]

9. Arruda-Olson AM, Olson LJ, Nehra A, Somers VK. Sleep apnea and cardiovascular disease. Implications for understanding erectile dysfunction. Herz 2003;28:298-303. [CrossRef]

10. Johns MW. A new method for measuring daytime sleepiness: the Epworth sleepiness scale. Sleep 1991;14:540-5.

11. Sleep-related breathing disorders in adults: recommendations for syndrome definition and measurement techniques in clinical research. The Report of an American Academy of Sleep Medicine Task Force. Sleep 1999;22:667-89.

12. Rechtschaffen A, Kales A. A Manual of Standardized Terminology, Technique, and Scoring System for Sleep Stages of Human Subjects, BIS/ BRI, UCLA, Los Angeles, 1968.

13. Rosen RC, Riley A, Wagner G, Osterloh IH, Kirkpatrick J, Mishra A. The international index of erectile function (IIEF): a multidimensional scale for assessment of erectile dysfunction. Urology 1997;49:822-30. [CrossRef]

14. McHorney CA, Kosinski M, Ware JE Jr. Comparisons of the costs and quality of norms for the SF-36 health survey collected by mail versus telephone interview: results from a national survey. Med Care 1994;32:551-67. [CrossRef]

15. Koçyigit H, Gülseren S, Erol A, Hizli N, Memis A. The reliability and validity of the Turkish version of Quality of Life Questionnaire of the European Foundation for Osteoporosis (QUALEFFO). Clin Rheumatol 2003;22:18-23. [CrossRef]

16. Akkus E, Kadioglu A, Esen A, Doran S, Ergen A, Anafarta K, et al. Prevalence and correlates of erectile dysfunction in Turkey: a population-based study. Eur Urol 2002;41:298-304. [CrossRef]

17. Physical status: the use and interpretation of anthropometry. Report of a WHO Expert Committee. World Health Organ Tech Rep Ser 1995;854:1-452.

18. Budweiser S, Enderlein S, Jörres RA, Hitzl AP, Wieland WF, Pfeifer $\mathrm{M}$, et al. Sleep apnea is an independent correlate of erectile and sexual dysfunction. J Sex Med 2009;6:3147-57. [CrossRef]
19. Fanfulla F, Malaguti S, Montagna T, Salvini S, Bruschi C, Crotti P, et al. Erectile dysfunction in men with obstructive sleep apnea: an early sign of nerve involvement. Sleep 2000;23:775-81.

20. Zias N, Bezwada V, Gilman S, Chroneou A. Obstructive sleep apnea and erectile dysfunction: still a neglected risk factor? Sleep Breath 2009;13:3-10. [CrossRef]

21. Findley LJ, Wilhoit SC, Suratt PM. Apnea duration and hypoxemia during REM sleep in patients with obstructive sleep apnea. Chest 1985;87:432-6. [CrossRef]

22. Sériès F, Cormier Y, La Forge J. Influence of apnea type and sleep stage on nocturnal postapneic desaturation. Am Rev Respir Dis 1990;141:1522-6.

23. Cohen DB. Dysphoric affect and REM sleep. J Abnorm Psychol 1979;88:73-7. [CrossRef]

24. Giles DE, Roffwarg HP, Schlesser MA, Rush A. Which endogenous depressive symptoms relate to REM latency reduction? J Biol Psychiatry 1986;21:473-82. [CrossRef]

25. Ware JC, Hirshkowitz M. Characteristics of penile erections during sleep recorded from normal subjects. J Clin Neurophysiol 1992;9:78-87. [CrossRef]

26. Bacon CG, Mittleman MA, Kawachi I, Giovannucci E, Glasser DB, Rimm EB. Sexual function in men older than 50 years of age: results from the health professional's follow-up study. Ann Intern Med 2003:139:161-8.

27. Moore P, Bardwell WA, Ancoli-Israel S, Dimsdale JE. Association between polysomnographic sleep measures and healthrelated quality of life in obstructive sleep apnea. J Sleep Res 2001;10:303-8. [CrossRef]

28. Flemons WW, Reimer MA. Development of a disease-specific health-related quality of life questionnaire for sleep apnea. Am J Respir Crit Care Med 1998;158:494-503.

29. Teloken PE, Smith EB, Lodowsky C, Freedom T, Mulhall JP. Defining association between sleep apnea syndrome and erectile dysfunction. Urology 2006;67:1033-7. [CrossRef]

30. Margel D, Cohen M, Livne PM, Pillar G. Severe, but not mild, obstructive sleep apnea syndrome is associated with erectile dysfunction. Urology 2004;63:545-9. [CrossRef]

31. Hanak V, Jacobson DJ, McGree ME, Sauver JS, Lieber MM, Olson EJ, et al. Snoring as a risk factor for sexual dysfunction in community men. J Sex Med 2008;5:898-908. [CrossRef] 